\title{
Effects of Sodium tanshinone IIA sulfonate on H9C2 cardiomyocyte injury induced by sodium arsenite
}

Heli Xu ${ }^{1}$, Xinsheng Zhang ${ }^{1}$, Mengyao Liu ${ }^{1}$, Jiayu Li ${ }^{1}$, Wenjing Dou ${ }^{1}$, Xiyan Wang ${ }^{1}$, Hongyou Tan ${ }^{1}$, Jian Ding ${ }^{1}$, Juhua Xie ${ }^{2}$, Yue Zhang ${ }^{1 *}$

1,Department of Occupational health and Occupationa Medicine, School of public health, Shenyang Medical College, Shenyang City, Liaoning Province

2. School of Basic Science, Shenyang Medical College

National innovation and entrepreneurship training project for college stud ents (201910164020); Shenyang high level Innovation Talents project (RC 190477);Liaoning provincial key R \& D project (2018225013); Shenyang Medical College Excellent Talents project (20113067); General project of National Natural Science Founding (81070128); Shenyang young and midd le-aged innovation support project (RC180379); Graduate student innovatio $\mathrm{n}$ funding project ofShen Yang Medical College (Y20190501, Y20190507) $\mathrm{y}$

*,Correspondence Author: Yue Zhang, e-mail: zhangyue2009@126.com

\begin{abstract}
Arsenic is a grade I human carcinogen that can cause kinds of damage to the body. The heart is one of the main target organs of arsenic damage, but the preventive and curative measures underlying arsenic poisoning are not clear. To investigate the effect of tanshinone IIA sulfonate (STS) on the injury of H9C2 cardiomyocytes induced by $\mathrm{NaAsO}$,H9C2 cardiomyocyteswere divided into four groups where control group with general medium,arsenic (As) group received sodium arsenite (NaAsO2)-containing general medium,STS pretreatment + As group and finally the STS alone group . Compared with the control group, different concentrations of STS had no significant effect on the cell viability $(\mathrm{n}=6, \mathrm{P}>0.05)$; Compared with the control group, there was no significant change in the viability of cells treated with arsenic for $12 \mathrm{~h}$ after STS pretreatment for $6 \mathrm{~h}(\mathrm{n}=6, \mathrm{P}>0.05)$, the viability of cells treated with $\mathrm{NaAsO} 2$ alone was decreased( $\mathrm{n}=6, \mathrm{P}<0.05)$;Compared with the control group, the cell viability was decreased after STS pretreatment for $12 \mathrm{~h}$ and arsenic for $24 \mathrm{~h}$, the cell viability of $\mathrm{NaAsO} 2$ group was also decreased,the viability of $\mathrm{NaAsO} 2$ cells was lower than that of STS pretreated for $12 \mathrm{~h}$ with $\mathrm{NaAsO} 2$ for $24 \mathrm{~h}(\mathrm{n}=6, \mathrm{p}<0.05)$. The content of Caspase $3 / 7$ in the $\mathrm{NaAsO} 2$ group was higher than that in the STS and $\mathrm{NaAsO} 2$ group and the Control group and STS group after 12h,the differences were statistically significant $(n=6, p<0.05)$.After STS pretreatment for $12 \mathrm{~h}$
\end{abstract}


and $\mathrm{NaAsO} 2$ for $24 \mathrm{~h}$, there was no significant difference in Caspase 8 content between $\mathrm{NaAsO} 2$ group and other groups $(\mathrm{n}=6, \mathrm{P}>0.05)$ As a result, Sodium tanshinone IIA sulfonate can attenuate the injury of $\mathrm{H} 9 \mathrm{C} 2$ cardiomyocytes induced by sodium arsenite.

Key Words: Sodium arsenite;Tanshinone IIA sulfonate sodium;H9c2 cardiomyocytes; apoptosis

\section{Introduction}

TANIIA is one of the components extracted from Salvia Miltiorrhiza Bunge, but it is not easily absorbed through intestinal tract.And for that,Sodium tanshinone IIA sulfonate (STS) was invented to improve its bioavailability.STS, which induces Vasodilation, inhibits inflammation, prevents arteriosclerosis, heart damage and hypertrophy, is considered to be a promising natural heart protective drug. ${ }^{1,2}$ Arsenic is a highly toxic and widely distributed metallic element that has been used to treat tumors for more than 2,000 years,Especially for Acute promyelocytic leukemia.However, long-term exposure to arsenic can lead to various diseases, especially to cardiovascular system. Arsenic can cause many kinds of cardiac damage, including electrophysiological changes and myocardial necrosis. ${ }^{3,4)}$ At present, there is not a good drug to antagonize the cardiac toxicity of arsenic, so this study is to investigate the effect of Sodium tanshinone IIA sulfonate on the injury of rat $\mathrm{H} 9 \mathrm{C} 2$ cardiomyocytes induced by $\mathrm{NaAsO} 2$, it provides a new way to prevent and treat the cardiac toxicity of arsenic.

\section{Materials and methods}

\section{Cells, Reagents.}

H9C2 rat cardiomyocyte strain(Shanghai Fumeng Gene Biotechnology company); DMEM Culture Medium(Hyclone company); FBS(Hyclone Corporation); Trypsin (Lifescience company); DPBS (Hyclone company); sodium arsenite (Analytical reagent, Fluka company); Tanshinone IIA sodium sulfonate(Shanghai Shifeng Biotechnology company); CCK-8 Kit (Dojindo company); caspase8 Kit (Promega company); Caspase3 / 7 Kit (Promega company).

\section{Cell culture.}

Rat $\mathrm{H} 9 \mathrm{C} 2$ cardiomyocytes were cultured in DMEM containing 10\% fetal bovine serum (FBS) at $37{ }^{\circ} \mathrm{C}$ with a volume fraction of $5 \% \mathrm{CO} 2$. The cells were digested with $0.25 \%$ trypsin and terminated with DMEM containing $10 \%$ FBS. The passage ratio was 1:3.

CCK-8 was used to detect the viability of cells treated with STS alone. 
The cells were inoculated into 96-well plates with 2000 cells / well, 6 samples in each group, then cultured in DMEM without serum for $12 \mathrm{~h}$. The cells were treated with $0 /$ $10 / 20 / 30 / 40$ / 50 / $100 \mathrm{uMol} / \mathrm{L}$ STS for $12 \mathrm{~h}$, then the cell viability was measured by CCK-8 Kit.

CCK-8 was used to detect the viability of cells pretreated with STS for 6h and treated with $\mathrm{NaAsO2}$ for $12 \mathrm{~h}$.

The cells were divided into control group 、 treated with $\mathrm{NaAsO} 2$ alone, the concentrations were $15 \mathrm{umol} / \mathrm{L}$ 、STS was pretreated for $6 \mathrm{~h}$ and $\mathrm{NaAsO} 2$ was added for 12h,the concentrations of STS were $10 / 20 / 30$ / 40 / 50umol/L,then the cell viability was measured by CCK-8 Kit.

CCK-8 was used to detect the viability of cells pretreated with STS for $12 \mathrm{~h}$ and treated with $\mathrm{NaAsO} 2$ for $24 \mathrm{~h}$.

The cells were divided into control group 、 treated with $\mathrm{NaAsO} 2$ alone and the concentrations were $10 \mathrm{umol} / \mathrm{L}$ 、STS was pretreated for $12 \mathrm{~h}$ and $\mathrm{NaAsO} 2$ was added for $24 \mathrm{~h}$,the concentrations of STS were $10 / 20 / 30 / 40 / 50 \mathrm{umol} / \mathrm{L}$, hen the cell viability was measured by CCK-8 Kit.

Caspase 3/7 was used to detect the apoptosis in the combination of STS and $\mathrm{NaAsO2.}$

The cells were divided into control group, NaAsO2 Group (15umol / L), STS group (20umol / L) , NaAsO2 group and STS combined group, and CASPASE3 / 7 was detected by CASPASE3 / 7 Kit after 8 hours.

Caspase 8 was used to detect the apoptosis of NaAsO2 after STS pretreatment.

The cells were divided into Control Group 、 NaAsO2 group (10umol / L) 、STS was pretreated for $12 \mathrm{~h}$ and $\mathrm{NaAsO} 2$ was added for $24 \mathrm{~h}$, the concentrations of STS were 10 / 20 / 30 / 40 / 50umol/L, Caspase8 kit was used to detect the content of Caspase8 in cells.

Statistical methods.

SPSS 21. 0 Software was used to analyze the data, and univariate Anova was used to test the significance of the inter-group comparison. For two comparisons, either LSD-t method or Dunnett's t 3 method was used when the homogeneity of the variance was satisfied. With a $\mathrm{p}<0.05$ was statistically significant.

\section{RESULTS}

\section{The effect of STS alone on cell viability.}

Compared with the control group, different concentrations of STS had no significant effect on the cell viability $(n=6, P>0.05), \quad$ see figure 1 . 


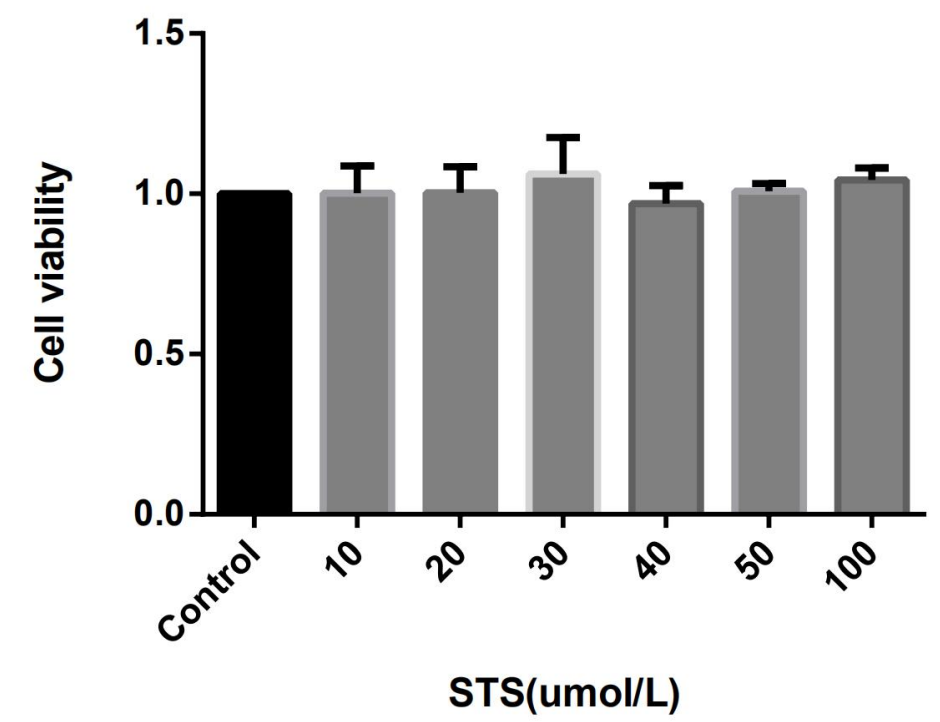

Fig 1: Effect of gradient concentration of STS on cell viability

The effect of $6 \mathrm{~h}$ after STS pretreatment and $12 \mathrm{~h}$ after NaAsO2 treatment on cell viability.

Compared with the control group, there was no significant change in the viability of cells treated with arsenic for $12 \mathrm{~h}$ after STS pretreatment for $6 \mathrm{~h}(\mathrm{n}=6, \mathrm{P}>0.05)$, the viability of cells treated with $\mathrm{NaAsO} 2$ alone was decreased( $\mathrm{n}=6, \mathrm{P}<0.05)$, see figure 2 .

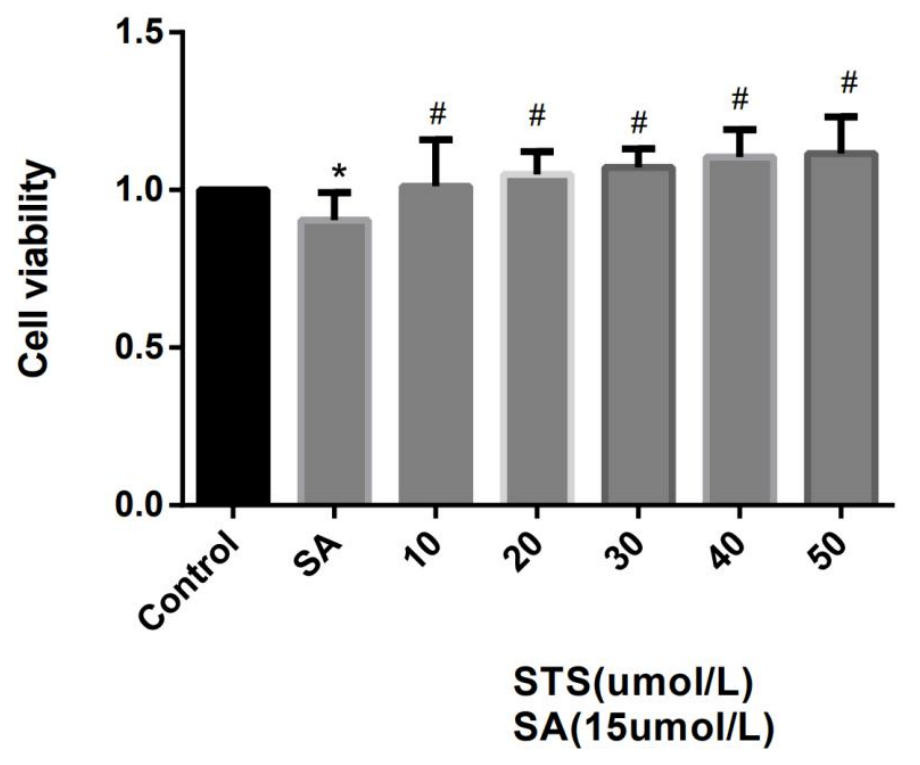

Fig 2: Changes of cell viability after STS pretreatment for 6 hours plus NaAsO2 12h

$\mathrm{n}=6$, Compared With Control Group, $* \mathrm{p}<0.05$

Compared with SA, \# $p<0.05$

The effect of $12 \mathrm{~h}$ after STS pretreatment and $24 \mathrm{~h}$ after $\mathrm{NaAsO2}$ treatment on cell viability. 
Compared with the control group, the cell viability was decreased after STS pretreatment for $12 \mathrm{~h}$ and arsenic for $24 \mathrm{~h}$, the cell viability of $\mathrm{NaAsO} 2$ group was also decreased,the viability of $\mathrm{NaAsO} 2$ cells was lower than that of STS pretreated for $12 \mathrm{~h}$ with $\mathrm{NaAsO} 2$ for $24 \mathrm{~h}(\mathrm{n}=6, \mathrm{p}<0.05)$, see figure 3

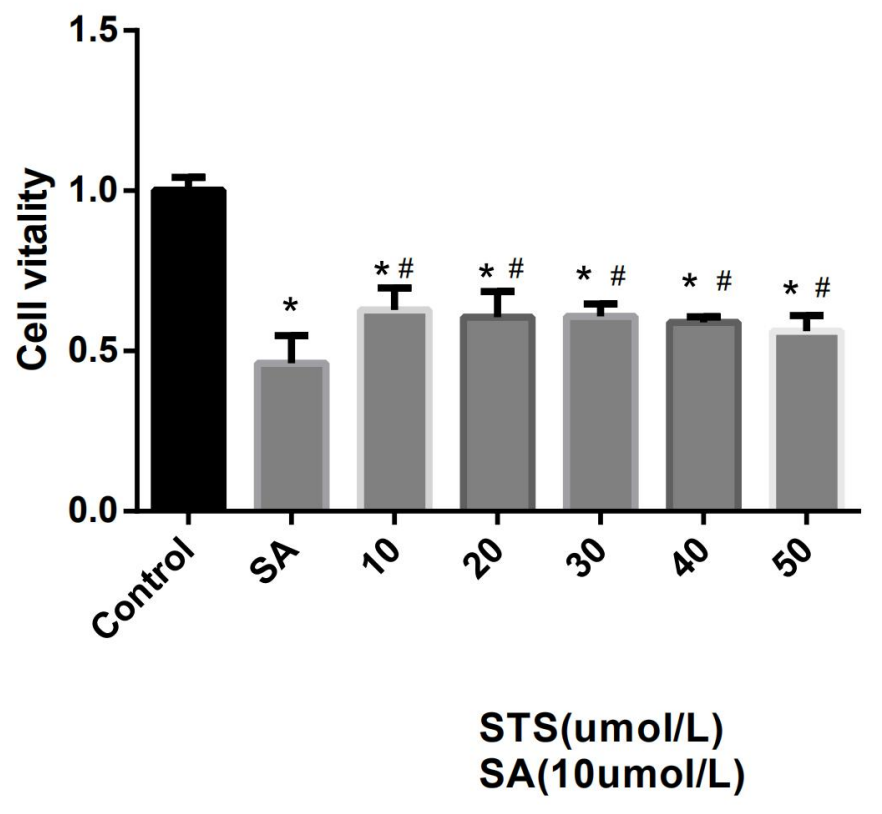

Fig 3: Changes of cell viability after STS pretreatment for 12 hours plus $\mathrm{NaAsO} 224 \mathrm{~h}$

Note: $\mathrm{n}=6$, Compared With Control Group, $* \mathrm{p}<0.05$

Compared with SA, \# $\mathrm{p}<0.05$

Changes of Caspase 3 / 7 in cells treated with combination of STS and NaAsO2.

The content of Caspase $3 / 7$ in the $\mathrm{NaAsO} 2$ group was higher than that in the STS and $\mathrm{NaAsO} 2$ group and the Control group and STS group after $12 \mathrm{~h}$, the differences were statistically significant $(\mathrm{n}=6, \mathrm{p}<0.05)$, see figure 4 . 


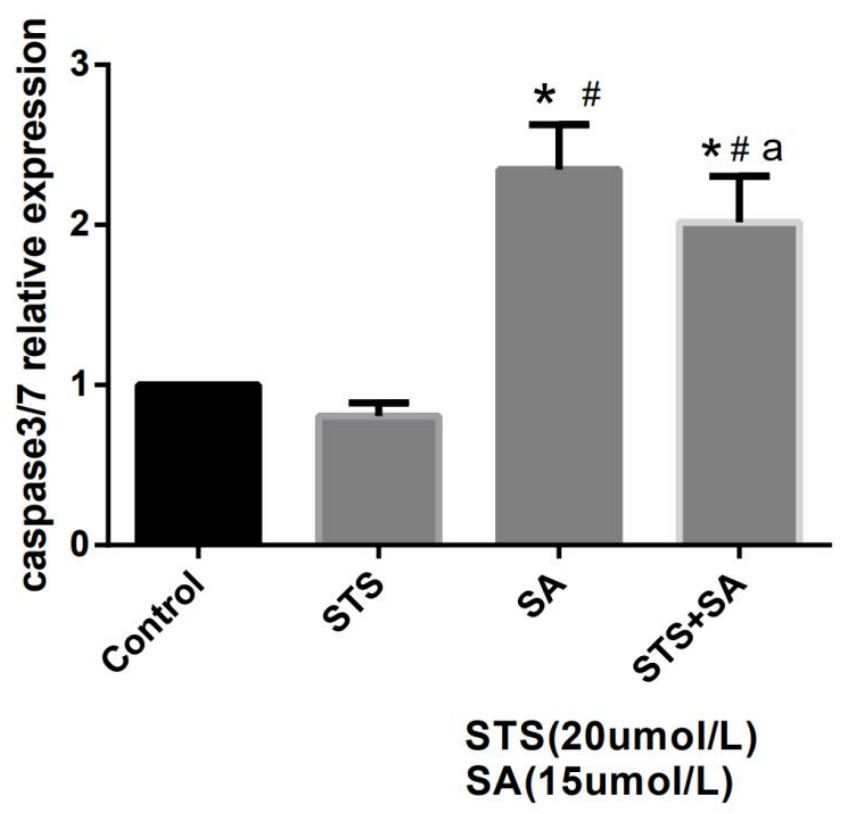

Fig 4: Relative Caspase 3 / 7 content after STS and NaAsO2 combined treatment

$\mathrm{n}=6 ; \quad$ Compared With Control Group, $* \mathrm{p}<0.05$

Compared with STS, \# p $<0.05$

Compared with $\mathrm{SA}, \mathrm{aP}<0.05$

Changes of Caspase 8 in cells with $12 \mathrm{~h}$ after STS pretreatment and $24 \mathrm{~h}$ after NaAsO2 treatment.

After STS pretreatment for $12 \mathrm{~h}$ and $\mathrm{NaAsO} 2$ for $24 \mathrm{~h}$, there was no significant difference in Caspase 8 content between $\mathrm{NaAsO} 2$ group and other groups $(\mathrm{n}=6, \mathrm{P}>0.05)$, see figure 5

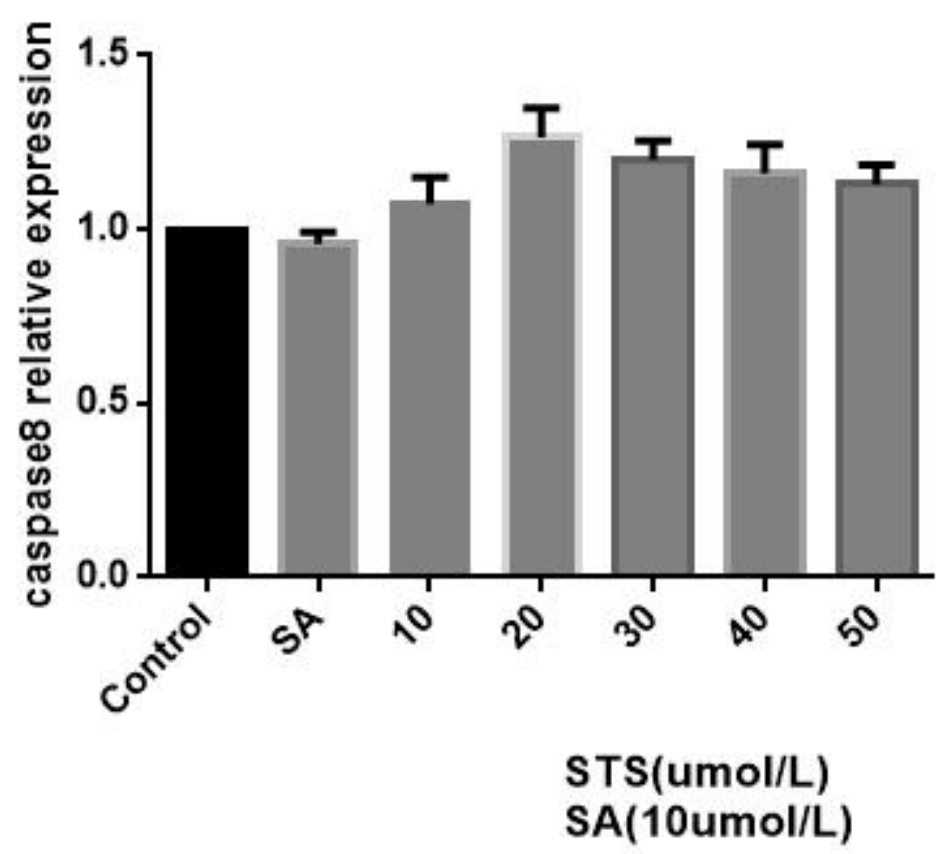

Fig 5. Caspase 8 relative content after STS pretreatment plus NaAsO2. 


\section{Discussion}

Arsenic is an ancient toxic metal that is commonly found in the environment. $\mathrm{NaAsO} 2$ is the most toxic, affecting people in many countries around the world, including China.Arsenic has been shown to cause many cardiovascular diseases and to cause cardiomyocyte damage and cardiomyocyte apoptosis ${ }^{5,6)}$. Sodium tanshinone IIA sulfonate (STS) has been approved by China Food and Drug Administration (CFDA) for the treatment of cardiovascular disease.$^{7,8}$ However, the combination of $\mathrm{NaAsO} 2$ poisoning and STS has not been reported. The effect of STS on myocardial injury induced by $\mathrm{NaAsO} 2$ was investigated at the cellular level. The results of CCK-8 Assay showed that A certain concentration of STS (0-50 and 100umol / 1) had no cytotoxic effect on H9C2 cells. In addition, STS pretreatment can effectively reverse the decline in cell viability caused by $\mathrm{NaAsO} 2$ and reduce the apoptosis of $\mathrm{H} 9 \mathrm{c} 2$ cardiomyocytes induced by sodium arsenite.

Caspase is a family of proteases that are closely related to apoptosis. They are mainly divided into initiating and executive types. Both are activated sequentially by foreign protein signals, leading to programmed cell death. The initial caspase- 8 is a molecular switch for apoptosis and necrosis, and caspase- 3 and -7 are related factors for performing apoptosis. Its overexpression has been shown to reduce myocardial contractility and cause heart disease in many pathological diseases.

Insufficiency. ${ }^{9.10,11,12)}$ Nagalakshmi Prasanna and other studies have confirmed that $\mathrm{NaAsO} 2$ administration can up-regulate caspase 3 in rat heart tissue. ${ }^{13}$ and the results of this study are in good agreement with it.. In addition, STS can inhibit the expression of caspase $3 / 7$ in the $\mathrm{NaAsO} 2$-treated group in this study, which also suggests that STS may protect myocardial cells from the apoptosis induced by sodium arsenite to a certain extent.In this study, sodium tanshinone IIA sulfonate could inhibit the increase of caspase-3 expression level, but there was no significant change in the expression level of caspase-8. Previous studies have shown that Bax / Bcl-2, caspase-3, and caspase-9 play important roles in the mitochondrial apoptosis pathway, while caspase- 8 and caspase- 12 are death receptor signaling pathways and endoplasmic reticulum apoptosis pathways, respectively. Key factors in. ${ }^{14}$ Therefore, the anti-apoptotic effect of tanshinone IIA sodium sulfonate may be mediated through a mitochondrial-dependent apoptosis pathway.

In short, the use of sodium tanshinone IIA sulfonate can reverse the decline in cell viability and increase of apoptosis induced by sodium 
arsenite, which provides a feasible method for the inhibition of sodium arsenone IIA sulfonate on apoptosis The molecular explanation provides a new idea for the prevention and treatment of sodium arsenite-induced heart failure. Further research is needed to elucidate the exact mechanism of the protective effect of sodium tanshinone IIA sulfonate on myocardial apoptosis.

\section{ACKNOWLEDGMENTS}

This work was supported in part by National Natural Science Foundation of China and Public Health Experimental Teaching Center of Shenyang Medical College.

\section{CONFLICT OF INTEREST}

All authors declare to have no actual or potential conflicts of interest

\section{REFERENCES}

1) Yu M L, Li S M, Gao X, et al. Sodium Tanshinone IIA Sulfonate for Coronary Heart Disease: A Systematic Review of Randomized Controlled Trials[J]. Chinese Journal of Integrative Medicine, 2018.

2) Tangting C, Miaoling L, Xuehui F, et al. Sodium Tanshinone IIA Sulfonate Prevents Angiotensin II-Induced Differentiation of Human Atrial Fibroblasts into Myofibroblasts[J]. Oxidative Medicine and Cellular Longevity, 2018, 2018:1-10. 3) Heng Wang, yangdan Ji, Yuesheng Yu, et al.. Protective effect of Catechin on myocardial toxicity induced by sodium arsenite [J]. Journal of Zunyi Medical College, 2018,41 (01): 33-37

4) Adil M , Kandhare A D, Ghosh P, et al. Sodium arsenite-induced myocardial bruise in rats: Ameliorative effect of naringin via TGF- $\beta / \mathrm{Smad}$ and $\mathrm{Nrf} / \mathrm{HO}$ pathways[J]. Chemico-Biological Interactions, 2016, 253:66-77.

5) Goudarzi M, Fatemi I , Siahpoosh A, et al. Protective Effect of Ellagic Acid Against Sodium Arsenite-Induced Cardio- and Hematotoxicity in Rats[J]. Cardiovascular Toxicology, 2018.

6) Phan N N, Li K L, Lin Y C . Arsenic induces cardiac rhythm dysfunction and acylcarnitines metabolism perturbation in rats $[\mathrm{J}]$. Toxicology Mechanisms and Methods, 2018:1-9.

7) $\mathrm{Wu} \mathrm{P}, \mathrm{Du} \mathrm{Y}, \mathrm{Xu} \mathrm{Z}$, et al. Protective effects of sodium tanshinone IIA sulfonate on 
cardiac function after myocardial infarction in mice. Am J Transl Res.

2019;11(1):351-360. Published 2019 Jan 15.

8) Zhou ZY, Zhao WR, Zhang J, et al. Sodium tanshinone IIA sulfonate: A review of pharmacological activity and pharmacokinetics. Biomed Pharmacother.

2019;118:109362. doi:10.1016/j.biopha.2019.109362

9) Fritsch M, Günther SD, Schwarzer R, et al. Caspase-8 is the molecular switch for apoptosis, necroptosis and pyroptosis. Nature. 2019;575(7784):683-687.

doi:10.1038/s41586-019-1770-6

10) Tummers B, Green DR. Caspase-8: regulating life and death. Immunol Rev. 2017;277(1):76-89. doi:10.1111/imr.12541

11) Cardona M, López JA, Serafín A, et al. Executioner Caspase-3 and 7 Deficiency Reduces Myocyte Number in the Developing Mouse Heart. PLoS One. 2015;10(6):e0131411. Published 2015 Jun 29. doi:10.1371/journal.pone.0131411 12) Erekat NS, Al-Jarrah MD. Association of Parkinson Disease Induction with Cardiac Upregulation of Apoptotic Mediators P53 and Active Caspase-3: An Immunohistochemistry Study. Med Sci Monit Basic Res. 2018;24:120-126. Published 2018 Aug 23. doi:10.12659/MSMBR.910307

13) Prasanna N, Rasool M. Modulation of gene-expression profiles associated with sodium arsenite-induced cardiotoxicity by p-coumaric acid, a common dietary polyphenol. J Biochem Mol Toxicol. 2014;28(4):174-180. doi:10.1002/jbt.21550 14) Wan CR, Han DD, Xu JQ, et al. Jujuboside A attenuates norepinephrine-induced apoptosis of H9c2 cardiomyocytes by modulating MAPK and AKT signaling pathways. Mol Med Rep. 2018;17(1):1132-1140. doi:10.3892/mmr.2017.7938 
bioRxiv preprint doi: https://doi.org/10.1101/2020.02.14.948505; this version posted February 15, 2020. The copyright holder for this preprint (which was not certified by peer review) is the author/funder. All rights reserved. No reuse allowed without permission. 